\title{
Incorporación de la cobertura de escape en un modelo de capacidad de carga para venado de cola blanca
}

\author{
Incorporation of escape cover in a model to estimate carrying capacity \\ for white-tailed deer \\ FX Plata ${ }^{a}$, JA Martínez ${ }^{a}$, GD Mendoza ${ }^{a}$, PA Hernández ${ }^{a}$, R Bárcena ${ }^{b}$, OA Villarreal ${ }^{*}$ \\ aDepartamento de Producción Agrícola y Animal, Universidad Autónoma Metropolitana, \\ Xochimilco, México. \\ bColegio de Postgraduados, Montecillo, México. \\ 'Benemérita Universidad Autónoma de Puebla, Puebla, México.
}

\begin{abstract}
SUMMARY
The objective of this study was to establish the incorporation of escape cover in a carrying capacity $(K)$ model for white-tailed deer (Odocoileus virginianus). The study was carried out in Santa Cruz Nuevo, Puebla, Mexico in an area of $50 \mathrm{Km}^{2}$. The information was gathered through 13 transects of 800 by six $\mathrm{m}$. The information served to establish the relationship between the escape cover and the faecal pellets of deer using a linear regression analysis. The relationship was included in a model for estimation of $K$, then a sensibility analysis was conducted. Faecal pellets were found in areas with an escape cover bigger than $59 \%$ and they were distributed in a cubic arrangement until reaching the $100 \%$. The sensibility analysis showed that the escape cover and the brush vegetation were the main components to increase the carrying capacity whereas the body weight of the deer reduces the $K$.
\end{abstract}

Palabras clave: modelos matemáticos, capacidad de carga, análisis de sensibilidad, venado cola blanca.

Key words: mathematical models, carrying capacity, sensibility analysis, white-tailed deer.

\section{INTRODUCCIÓN}

La capacidad de carga es el número máximo de individuos de una población que puede contener un hábitat determinado en un área definida, la cual se delimita por restricciones como la disponibilidad de espacio, agua y biomasa comestible (Gotelli 2008). Esta definición explica los cambios en el tamaño y distribución de algunas poblaciones (Bevers y Flather 1999). Una estimación adecuada de la $K$ es fundamental para contribuir al mantenimiento del equilibrio entre la población de animales silvestres y la comunidad vegetal y permite evaluar el impacto de la pérdida o alteraciones de éste en el tamaño de una población (Cook y col 2007). Dicha estimación puede ser realizada utilizando diferentes modelos, dentro de los cuales se encuentran los índices de calidad del hábitat (Short 1986, Brooks 1997), a través del establecimiento del punto de equilibrio entre la tasa de crecimiento de una población y el tamaño de la misma en el área (Mandujano y col 2007) o a partir de la disponibilidad de alimentos o nutrientes específicos requeridos por una especie animal dada (McLeod 1997).

Debido a que la disponibilidad de nutrientes garantiza un crecimiento adecuado de la población y una alta tasa

Aceptado: 29.11.2012.

* Mazamiztli@yahoo.com.mx de sobrevivencia de los animales silvestres, estos modelos han sido evaluados en diversas especies animales (Hobbs y Swift 1985, DeYoung y col 2000, Brasher y col 2007, Beck y col 2006), utilizando la materia seca, nitrógeno y energía digestible como los principales nutrientes vegetales disponibles en el área y comparándolos versus los requerimientos nutricionales de la especie evaluada.

Después de la alimentación, dos de los componentes más importantes del hábitat son la presencia de agua y la cobertura. Esta última variable se define como el elemento estructural del hábitat, el cual alberga, oculta y protege a los animales en vida libre. Dicha variable puede ser clasificada de diversas formas, pero se acepta que la cobertura de escape es la que le permite al animal esconderse de los depredadores y reducir el riesgo de ataques (Mysterud y Østbye 1999). En los venados se ha encontrado que gran parte del tiempo que utilizan para el descanso lo realizan en áreas con una gran cobertura de este tipo; mientras que para su alimentación utilizan áreas más abiertas pero en las cuales el contenido nutricional de las plantas es más alto (Pollock y col 1994, Germaine y col 2004). En las zonas áridas, estos cambios en la distribución de la vegetación son el resultado de asociaciones vegetales que les permiten aumentar la probabilidad de supervivencia a las plantas y debido a las altas temperaturas ambientales que se presentan en estas regiones, la temperatura del suelo aumenta, lo que ocasiona que las especies vegetales crezcan en pequeños microhábitats que no son simétricos (Peters y col 2008). 
Uno de los problemas que tienen los modelos para estimación de la $K$ utilizando la disponibilidad de nutrientes para el hábitat del venado de cola blanca (Odocoileus virginianus) es que no estiman adecuadamente la capacidad del mismo (Hobbs y Swift 1985, McCall y col 1997, Plata y col 2011 a). Debido a que estos modelos sólo consideran la disponibilidad de nutrientes, la falta de exactitud de los mismos puede ser debida a que no consideran otros factores como la distribución de las especies vegetales, la presencia del agua y la existencia de cobertura de escape que les permite esconderse de los depredadores.

Considerando lo anterior, los objetivos de este trabajo fueron caracterizar la forma de la distribución de la vegetación, estimar el impacto de la cobertura de escape e incorporarla a un modelo de capacidad de carga de tipo nutricional. Luego, evaluar mediante un análisis de sensibilidad el impacto de los componentes del modelo en la capacidad de carga de un área determinada donde habita el venado de cola blanca.

\section{MATERIAL Y MÉTODOS}

\section{ÁREA DE ESTUDIO}

El trabajo se realizó con información obtenida en un rancho cinegético con una superficie de 5.309,44 ha, localizado en la comunidad de Santa Cruz Nuevo (18²0’ N y 9751' W), Municipio de Totoltepec de Gro, Puebla, México. Predominando los climas semicálido subhúmedo con lluvias en verano (A)C(wo), con temperatura media anual de 22 a $25^{\circ} \mathrm{C}$; y precipitación pluvial anual de 759 a $950 \mathrm{~mm}$ y templado subhúmedo con lluvias en verano $\mathrm{C}$ (wo), con temperatura media anual de 17 a $19^{\circ} \mathrm{C}$; y precipitación pluvial de 650 a $700 \mathrm{~mm}$. La pendiente va del 20 al $70 \%$ de inclinación y suelo somero de 0 a 25 cm (Arriaga y col 2000). Los tipos de vegetación son cuatro: 1) selva baja caducifolia, en algunas partes este tipo de vegetación se presenta como un matorral arborescente, debido a la falta de humedad y pobreza del suelo, 2) matorral mediano subinerme. 3) zonas de encinos que se presentan en las partes cerriles más altas y 4) selva mediana subcaducifolia (Rzedowski 2006).

\section{DISTRIBUCIÓN Y DENSIDAD DE ESPECIES VEGETALES}

Con la finalidad de determinar las características de la distribución de la vegetación en el área de estudio, se realizó un muestreo de caracterización en 13 sitios elegidos al azar, los cuales fueron estratificados en base a su altura sobre el nivel del mar. Dentro de estos sitios se marcaron cuadros de 15 x 15 m (Bullock 1999). Se estimó la diversidad y la riqueza de la vegetación y se utilizó el índice de Shannon (Buckland y col 2005) como una medida de relación. Previo al análisis estadístico y para determinar la factibilidad de la utilización del procedimiento GLM del SAS, se analizó la forma de distribución de las especies vegetales utilizando la prueba de Shapiro Wilk (Distribución normal) y Cramer-von Mises (Distribución Weibull). Debido a que la prueba de Shapiro Wilk fue diferente estadísticamente $(\mathrm{P}<0,001)$ y para evaluar el efecto de la ASNM en la distribución de la vegetación se realizó una prueba de Kruskal-Wallis utilizando dos altitudes; una de los 1.550 a los $1.700 \mathrm{~m}$ y otra de los $1.700 \mathrm{~m}$ en adelante. Todas las pruebas se realizaron utilizando el programa JMP 7 (Sall y col 2007).

BIOMASA VEGETAL TOTAL

Con la finalidad de establecer la relación entre la biomasa vegetal, la cobertura de escape y la presencia de fecas de venado cola blanca, se establecieron 13 cuadros de $5 \mathrm{x}$ $10 \mathrm{~m}$, los cuales fueron subdivididos en cuatro cuadrantes. Estos se localizaron a menos de $2.200 \mathrm{~m}$ de distancia de los aguajes permanentes. Dentro de estos cuadrantes se identificaron y contaron todas las arbustivas y arbóreas presentes. Para determinar la biomasa (kg/ha) en estos tipos de vegetación y debido a que la mediana y la moda del número de individuos de cada especie vegetal dentro del cuadro eran 4 y 1 respectivamente, se seleccionaron aleatoriamente dos ejemplares de las mismas presentes en el cuadro primario. Se cortaron las hojas y flores jóvenes de las ramas siguiendo los métodos descritos por Schuster (1965) para arbóreas y Telfer (1969) para arbustivas respectivamente. El promedio de la biomasa de ambas plantas se multiplicó por el número total de las mismas dentro del cuadro y el resultado se extrapoló a kg/ha. Para el caso de las arbustivas y arbóreas con una altura mayor a 1,60 m solo se consideraron las ramas y la biomasa disponible por debajo de esta altura. La biomasa de las gramíneas y herbáceas se estimó siguiendo la técnica propuesta por Williamson y col (1987).

\section{BIOMASA VEGETAL UTILIZABLE POR EL VENADO COLA} BLANCA

Se realizaron dos estimaciones empleando los valores obtenidos de la biomasa vegetal total. La primera, se calculó de forma tradicional con un coeficiente de utilización del 35\% (Stuth y Sheffield 2001) y en la segunda se utilizó la adecuación de la estimación de la biomasa de gramíneas en función de las arbóreas y arbustivas siguiendo las modificaciones sugeridas por Plata y col $\left(2011^{\mathrm{b}}\right)$. Con la finalidad de conocer si existen diferencias entre dichos métodos de estimación, se efectuó un análisis de varianza con un diseño completamente al azar con 13 repeticiones por tratamiento utilizando el programa JMP 7 (Sall y col 2007).

GENERACIÓN DEL FACTOR DE CORRECCIÓN POR COBERTURA DE ESCAPE

Para establecer la relación entre la cobertura de escape (ce) y la presencia del venado, se determinó el número de 
fecas y la ce presentes a lo largo de los transectos cuyo origen estuvo en el cuadro primario utilizado para la estimación de la biomasa vegetal. Los transectos tuvieron una longitud de 600-800 m por $6 \mathrm{~m}$ de ancho. Se consideró la ce como el porcentaje de vegetación capaz de ocultar a un sujeto cuando se encuentra a $15 \mathrm{~m}$ de distancia. Para estimarla, se utilizó un estadal de 2,00 m de largo marcado con colores alternos (rojo y blanco) cada $10 \mathrm{~cm}$, el cual fue colocado a $15 \mathrm{~m}$ del observador. El número de cuadros cubiertos por debajo de 1,5 m de altura fue expresado como porcentaje y la ce del sitio fue el promedio de las lecturas de los cuatro puntos cardinales (Griffith y Youtie 1988). Esta variable se midió al inicio y al final de cada $100 \mathrm{~m}$ del transecto. La cobertura promedio de todos los puntos del transecto se empleó para establecer el valor de cambio del número de fecas. El valor de cambio en este número, con respecto a la cobertura de escape, se estimó mediante un análisis de regresión lineal; el intercepto y la pendiente de la regresión fueron realizados con el programa JMP7 de SAS (Sall y col 2007).

\section{INCORPORACIÓN DE COBERTURA DE ESCAPE EN EL MODELO}

El modelo que se modificó con la cobertura de escape fue el empleado por Plata y col (2011 a), en donde se estimaba la $K$ de un sitio a partir de la energía metabolizable de los diversos grupos vegetales que se encontraban presentes en el sitio (1). Utilizando la modificación propuesta por estos mismos autores (Plata y col 2011 b) para condicionar la utilización de las gramíneas a la disponibilidad de las arbustivas y las arbóreas (2). Dicha incorporación generó la siguiente ecuación (3).

$$
K=\frac{\sum_{1 \ldots . . n} E m f * A * 0,35}{\sum_{1 \ldots m} M E V_{d}}
$$

Donde: $K$ es capacidad de carga (venados de $60 \mathrm{~kg} / \mathrm{ha}$ ).

Emf = Energía metabolizable del forraje de la especie vegetal 1 hasta $\mathrm{n}(\mathrm{kcal} / \mathrm{ha})$.

$A \quad=$ Área total del predio (ha).

$n \quad=$ Estrato.

$m \quad=$ Día juliano.

$M E V_{d}=$ Metabolismo ecológico diario de un venado de $60 \mathrm{~kg}$ (kcal/día).

0,35 = Eficiencia de utilización del forraje.

$$
E M g=\left(\frac{B u * 0,08 * A * B g * A}{B g * A}\right) * E B g * D I V M S g * 0,82
$$

Donde:

EMg = Energía metabolizable de las gramíneas (Mcal/ kg MS).

$\mathrm{Bu}=$ Biomasa útil (biomasa de arbóreas $\mathrm{y}$ arbustivas) (kg/ha).
0,08 = Coeficiente de utilización de las gramíneas $(\%)$.

$B g \quad=$ Biomasa de gramíneas $(\mathrm{kg} / \mathrm{ha})$.

$A=$ Área (has).

$E B g=$ Energía bruta de las gramíneas (Mcal/kg).

$D I V M S g=$ Digestibilidad in vitro o in situ de la materia seca (MS) de las gramíneas.

0,82 = Valor de cambio de Energía digestible a energía metabolizable.

$$
K=\frac{\sum_{1 \ldots n} E m_{f u}+E m g * A * 0,35}{\sum_{1 \ldots m} M E V_{d}}
$$

$K=$ Capacidad de carga (venados/ha).

$E M_{f u}=$ Energía metabolizable del forraje útil (energía proveniente de arbóreas y arbustivas) ( $\mathrm{kcal} / \mathrm{kg} /$ ha).

Emg = Energía metabolizable de gramíneas $(\mathrm{kcal} / \mathrm{kg} /$ ha).

A = Área del hábitat evaluado (has).

0,35 = Eficiencia de utilización del forraje.

$M E V_{p}=$ Metabolismo en vida libre de un venado durante el período $p$.

Para este trabajo se sustituyó la energía metabolizable por biomasa vegetal y el metabolismo en vida libre de un venado se reemplazó por la capacidad máxima de consumo de fibra detergente neutro (FDN) de acuerdo a Mertens (1987) considerando que la capacidad de consumo de fibra era igual al 0,011 del peso vivo del animal, además se incluyó el factor de corrección por cobertura de escape (4).

$$
K=\frac{\sum_{1 \ldots n} B u+B g * A * 0,35 * C_{c \mathrm{e}}}{\sum_{1 \ldots m} R m s V_{p}}
$$

$K=$ Capacidad de carga (venados/ha).

$B_{u} \quad=$ Biomasa útil $(\mathrm{kg} / \mathrm{ha}) 1$ hasta $\mathrm{n} ; \mathrm{n}=$ grupos vegetales (arbóreas y arbustivas).

$B g \quad=$ Biomasa disponible de gramíneas $(\mathrm{kg} / \mathrm{ha})$.

$$
B g=B u * 0,08
$$

Dicho valor se aplica si la disponibilidad de gramíneas es mayor a la biomasa de la sumatoria de las arbustivas y las arbóreas. Si es menor entonces:

$$
B g=B_{t g} * 0,35(\mathrm{~kg} / \mathrm{ha})
$$

Donde:

$B_{t g}=$ Biomasa total de gramíneas en el área.

$0,35=$ Eficiencia de utilización del forraje.

$A=$ Área del hábitat (has).

$R m s V_{p}=$ Consumo máximo de biomasa en función de FDN y peso vivo del animal (Mertens 1987). 


$$
R m s V_{p}=\frac{0,011 * P V}{F D N_{R}}
$$

Donde:

$F D N_{\mathrm{R}}=\% \mathrm{FDN} / 100$.

$C_{c e} \quad=$ Corrección por cobertura de escape.

Donde:

$C_{c \mathrm{e}}=\mathrm{I} C \frac{I}{19,05118}$

I = Ecuación generada en este trabajo.

\section{ANÁLISIS DE SENSIBILIDAD}

Se realizó un análisis de sensibilidad con el modelo modificado siguiendo la técnica descrita por Bossel (1994). Donde la variación en los datos se analiza individualmente, mediante la modificación de un valor a la vez, asumiendo que todos los demás permanecen sin alteración alguna. Para la generación de los intervalos mínimos y máximos de cada variable se utilizaron los valores observados en los muestreos de campo descritos previamente. El valor de cambio de cada variable fue contrastado y discutido en términos absolutos.

\section{RESULTADOS Y DISCUSIÓN}

\section{DISTRIBUCIÓN Y DENSIDAD DE ESPECIES VEGETALES}

Se puede observar que para los datos de este estudio el número total de plantas por ha, la riqueza de especies y el índice de Shannon (cuadro 1) se ajustan mejor a una distribución de Weibull con tres parámetros. Esta distribución se caracteriza por ser asimétrica con respecto a la media (Sarkkola y col 2004), lo que significa que la probabilidad de encontrar áreas con mayor y menor vegetación con relación a esta variable no es proporcional. También se caracteriza por depender de tres parámetros $\alpha, \lambda$ y $\beta$ donde los últimos dos definen la forma y la escala de la distribución, de tal manera que se puede modelar la asimetría generada por el índice de Shannon (Rouvinen y Kuuluvainen 2005).
Estos cambios en las características de distribución de la vegetación son explicados por los cambios en la presencia o ausencia de especies en función de las características específicas de las microrregiones que conforman el área de estudio (Huerta-Martínez y col 2004). El crecimiento vegetal dentro de estas áreas es modificado por el relieve y el tipo de suelo, ocasionando relaciones de sinergia y competencia entre las especies vegetales (Austin 1999, Otto y col 2001, Peters y col 2008). Bajo las condiciones de este trabajo, la distribución de la vegetación tiende $\left(\mathrm{X}^{2}\right.$; $\mathrm{P}<0,11$ ) a ser mayor en las áreas más altas (cuadro 1), lo cual puede ocasionar cambios en la vegetación que afectan la cobertura de escape. Trabajos realizados por Long y col (2005) muestran que la tasa de dispersión del venado de cola blanca aumenta cuando la cobertura vegetal vertical se reduce, observándose lo mismo en corzos y en alces (Jiang y col 2010). Lo anterior puede explicar la ausencia de fecas del venado cola blanca en áreas con menor ASNM y baja cobertura de escape.

\section{BIOMASA VEGETAL UTILIZABLE POR EL VENADO}

La prueba de Kruskal-Wallis reveló que el estimado de biomasa utilizable por el venado depende del método usado (cuadro 2). La importancia del método de estimación de biomasa útil para el venado ha sido justificada desde los inicios del siglo pasado, cuando el Departamento de Agricultura de los Estados Unidos comenzó a reducir la utilización de pastizales por el ganado para promover el desarrollo de venados y como consecuencia de una sobreutilización de las áreas aumentó la mortalidad de los mismos y se afectó la cobertura vegetal (Young 1998).

Stuth y Sheffield (2001) muestran que reducir la utilización de la biomasa potencialmente disponible entre el 30 y $50 \%$ mejora la condición corporal de los venados cola blanca. Por su parte, Galt y col (2000) han discutido la conveniencia de utilizar el $25 \%$ del forraje en lugar del $35 \%$ habitual. La importancia de reducir el coeficiente de utilización en función de los hábitos de consumo permite disminuir el riesgo de sobrestimar la capacidad de carga de un hábitat y mejorar la condición de todas las especies presentes en el mismo. Debido a que el venado es una

Cuadro 1. Tipo de distribución para el número de plantas, riqueza y biodiversidad de las UMAS Santa Cruz Nuevo, Puebla, México. Distribution type for number of plants, richness and biodiversity from the UMAS in Santa Cruz Nuevo UMAS, Puebla, Mexico.

\begin{tabular}{|c|c|c|c|c|c|c|}
\hline & \multirow{2}{*}{$\begin{array}{c}\text { Tipo de } \\
\text { distribución }\end{array}$} & \multicolumn{2}{|c|}{ ASNM } & \multirow{2}{*}{$\mathrm{DE}$} & \multirow{2}{*}{$\mathrm{X}^{2}$} & \multirow{2}{*}{$\mathrm{P}<\mathrm{X}^{2}$} \\
\hline & & 1700 & 1600 & & & \\
\hline Número de plantas por ha & WB* & 243,25 & 173,28 & 3705 & 2,44 & 0,11 \\
\hline $\mathrm{N}^{o}$ de especies & WB* & 15,25 & 13,28 & 161,57 & 0,66 & 0,41 \\
\hline Índice de Shannon & WB* & $-1,72$ & $-1,59$ & 17,62 & 0,013 & 0,90 \\
\hline Biomasa total, $\mathrm{kg}$ & WB* & 118,4 & 167,9 & 6994, & 0,48 & 0,48 \\
\hline
\end{tabular}

* Normal (Shapiro Wilks) P > 0,005; Weibull tres parámetros; (Cramer-von Mises) $\mathrm{P}<0,15$. 
especie que prefiere consumir arbustivas y arbóreas a las gramíneas (Stuth y Sheffield 2001), reducir el coeficiente de utilización de las gramíneas evitará una sobrestimación de la $K$ del hábitat del venado cola blanca.

INCORPORACIÓN DEL FACTOR DE COBERTURA DE ESCAPE $\left(C_{C E}\right)$

La ecuación generada con la información sobre la presencia de fecas animales y la cobertura de escape (ce) se presenta en el cuadro 3. Trabajos realizados en climas áridos muestran que los componentes principales que determinan la presencia del venado son la altura de la vegetación, volumen vegetal, cobertura vertical y cobertura de escape (Gallina y col 2010). Aunque en este trabajo hubo sitios con poca cobertura donde se localizaron algunos indicios, la ecuación generada establece que la cobertura mínima requerida para poder encontrar fecas de venado fue del $56 \%$. DePerno y col (2002) caracterizaron los diferentes tipos de cobertura que utiliza el venado cola blanca y reportando que la cobertura de escape donde habitan los venados va a depender del tipo de actividad que realizan en ella, de tal manera que cuando se alimentan emplean una cobertura de escape más baja que cuando están echados ( 32,8 vs 53\%); también, Avey y col (2003) estimaron el área compartida entre el venado bura (Odocoileus hemiounus) y cola blanca en Texas, EUA. Sus resultados mostraron que la pendiente y el porcentaje de cobertura arbustiva representan la mayor parte de la varianza de la distribución de dichas especies. La media en la cobertura arbustiva utilizada por venados bura y venados cola blanca en áreas de descanso fue de $37,7 \%$ vs $53,9 \%$, respectivamente, lo cual coincide con los resultados de este trabajo.

\section{ANÁLISIS DE SENSIBILIDAD}

El análisis de sensibilidad (cuadro 4) mostró que los cambios en la biomasa proveniente de las arbustivas y arbóreas en forma conjunta modifican en forma sustancial la $K$ estimada por el modelo. La elección de las arbustivas como grupo vegetal para estimar $K$ está fundamentada en los resultados obtenidos por Gallina (1993) y Plata y col (2009). Quienes mostraron que en condiciones de disponibilidad alta o similar de biomasa, el venado cola blanca prefiere a las arbóreas y arbustivas sobre las herbáceas y gramíneas. Aunque existen reportes de la composición de la dieta que establecen que estos grupos vegetales no son los únicos componentes consumidos por el venado (Bryant y col 1981, Stuth y Sheffield 2001), ya que el venado tiende a seleccionar las especies o dietas con el mayor contenido nutricional (Bryant y col 1980, Berteaux y col 1998), tal es el caso de las arbustivas y las arbóreas que presentan un mejor contenido nutricional que las gramíneas (Codron y col 2007).

Cuadro 2. Estimación de la biomasa vegetal total y disponible de acuerdo al estrato para el venado cola blanca en las UMAS de Santa Cruz Nuevo, Puebla.

Total and available plant biomass estimation according to the strata for white-tailed deer in the UMAS of Santa Cruz UMA Nuevo, Puebla.

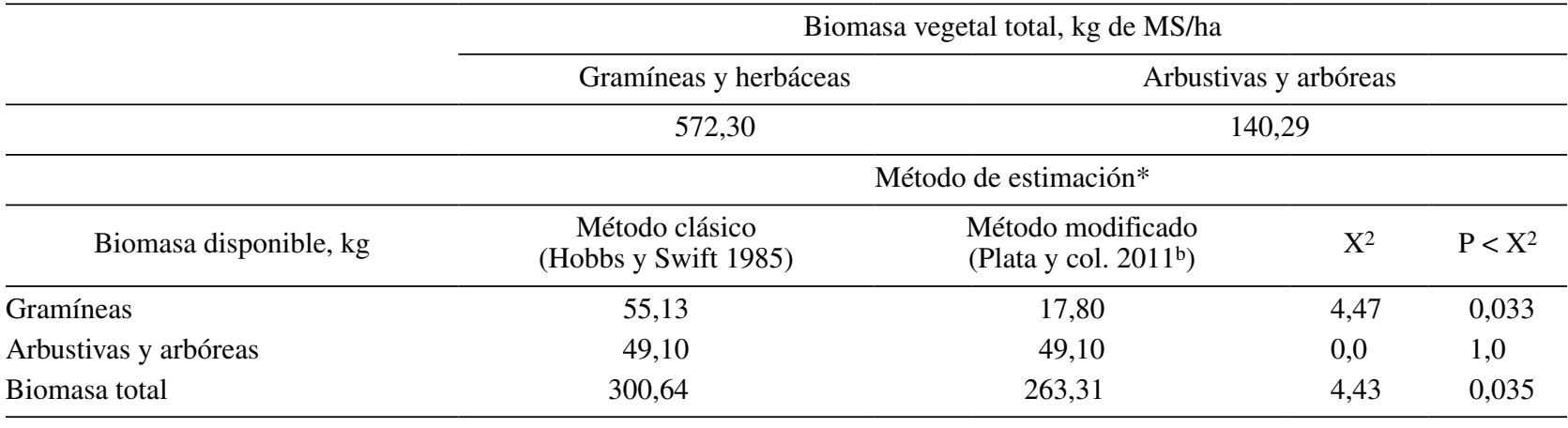

*Prueba de Kruskal-Wallis.

Cuadro 3. Análisis de regresión para estimar los valores de cambio del número de fecas de venado de cola blanca con respecto a la cobertura de escape.

Regression equation to estimate the change values for animal traces regarding escape cover.

\begin{tabular}{lcc}
\hline Ecuación & $\mathrm{R}^{2}$ & $\mathrm{P}$ \\
\hline $\mathrm{I}=-61,53247+110,68574 * \mathrm{CE}-134,64085 *(\mathrm{CE}-0,70569)^{2}-723,33447 *(\mathrm{CE}-0,70569)^{3}$ & 0,75 & 0,004 \\
\hline
\end{tabular}

$\mathrm{I}=$ Fecas de venado de cola blanca.

$\mathrm{CE}=$ Cobertura de escape. 
Cuadro 4. Análisis de sensibilidad de los componentes principales del modelo. Sensitivity analysis for the main components of the model.

\begin{tabular}{lcccc}
\hline Variable & Máximo & Mínimo & Intervalo & $\begin{array}{c}\text { Modelo } \\
\text { Modificado }\end{array}$ \\
\hline Arbustivas y arbóreas, kg MS/ha & & & & $\Delta K^{1}$ \\
Gramíneas y herbáceas, kg MS/ha & 303,6 & 3,6 & 50 & 0,348 \\
MS total kg/ha & 300 & 0 & 50 & 0,0019 \\
Cobertura de escape, \% & 961,37 & 361,37 & 100 & 0,13 \\
Peso vivo, kg & 1 & 0,59 & 0,07 & 1,81 \\
& 75 & 15 & 10 & $-0,19$ \\
\hline
\end{tabular}

MS = materia seca

En contraste con los modelos tradicionales, con el modelo generado en este trabajo no modifica la $K$, en función de la biomasa aportada por las gramíneas ni por la biomasa total del hábitat, lo cual es explicado por las restricciones de uso de las gramíneas como función de disponibilidad de las arbóreas y las arbustivas. Dicha restricción puede ser justificada por la naturaleza selectiva del venado, el cual tiene la habilidad de sustituir el tipo de arbustivas y herbáceas que consume a lo largo del año, sin necesidad de consumir pastos (Gallina 1993), aunado a la habilidad de los herbívoros de modificar su comportamiento alimenticio cuando el hábitat cambia (Gordon y col 2004).

El análisis de sensibilidad también reveló que la cobertura de escape tiene un papel importante dentro del modelo para modificar la $K$. Esto coincide con lo reportado por DePerno y col (2002) quienes muestran que los componentes principales que modifican la distribución del venado dentro del hábitat son la cobertura de escape, obstrucción visual y densidad de la vegetación, los cuales pueden explicar en gran medida la varianza de la distribución de esta especie dentro de un sitio dado.

El peso vivo del animal muestra que el valor de cambio en el modelo modificado es mayor que en el modelo nutricional en un $\Delta K$ de 0,7 . Dicha diferencia es debida a que el modelo nutricional utiliza el requerimiento de Galbraith y col (1998) para MS, mientras que el modelo modificado utiliza las ecuaciones de Mertens (1987). De acuerdo con Bergman y col (2001) las principales restricciones al consumo de energía en los ungulados proviene de la interacción entre sus limitantes físicas y fisiológicas y la interacción con las características estructurales del alimento que consumen de tal forma que uno de los principales reguladores del consumo de alimento es el llenado que se produce a nivel ruminal debido a la concentración de paredes celulares que contiene el alimento.

\section{RESUMEN}

Los objetivos de este trabajo fueron evaluar la incorporación de la cobertura de escape en un modelo de capacidad de carga $(K)$ de venado cola banca, evaluar el modelo por análisis de sensibilidad y comparar la estimación de la $K$ con el modelo versus un modelo basado en disponibilidad de nutrientes. El trabajo se desarrolló en la localidad de Santa Cruz Nuevo, Puebla, México, en un área de $50 \mathrm{~km}^{2}$, donde se establecieron 13 transectos de $800 \mathrm{~m}$ de longitud por 6 de ancho y se colectó información para establecer la relación entre la cobertura de escape y la presencia de indicios de venado mediante regresión lineal. Dicha relación se incorporó en un modelo de estimación de $K$, después de lo cual se realizó un análisis de sensibilidad del mismo. Los indicios animales se observaron principalmente en las áreas con una cobertura de escape mayor al 59\% y se distribuyeron en una forma cuadrática hasta llegar al 100\%. El análisis de sensibilidad mostró que la cobertura de escape y la vegetación arbustiva fueron los principales componentes para aumentar la capacidad de carga, mientras que el peso vivo del venado para disminuirla.

\section{REFERENCIAS}

Arriaga L, JM Espinoza, C Aguilar, E Martínez, L Gómez, E Loa. 2000. Regiones terrestres prioritarias de México. Comisión Nacional para el Conocimiento y Uso de la Biodiversidad, México, Pp 472-475.

Austin MP. 1999. The potential contribution of vegetation ecology to biodiversity research. Ecography 22, 465-484.

Avey JT, WB Ballard, MC Wallace, MH Humphrey, PR Krausman, F Harwell, EB Fish. 2003. Habitat relationships between sympatric mule deer and white-tailed deer in Texas. The Southwestern Naturalist 48, 644-653.

Beck JL, JM Peek, EK Strand. 2006. Estimates of elk summer range nutritional carrying capacity constrained by probabilities of habitat selection. J Wild Manage 70, 283-294.

Bergman CM, JM Fryxell, CC Gates, D Fortin. 2001. Ungulate foraging strategies: Energy maximizing or time minimizing? J Anim Ecol 70, 289-300.

Berteaux D, M Crête, J Huot, J Maltais, J Ouellet. 1998. Food choice by white-tailed deer in relation to protein and energy content of the diet: A field experiment. Oecologia 115, 84-92.

Bevers M, CH Flather. 1999. The distribution and abundance of populations limited at multiple spatial scales. J Anim Ecol 68, 976-987.

Bossel H. 1994. Modelling and Simulation. A.K. Peters Ltd., USA.

Brasher MG, JD Steckel, RJ Gates. 2007. Energetic carrying capacity of actively and passively managed wetlands for migrating ducks in Ohio. J Wild Manage, 71, 2532-2541.

Brooks RP.1997. Improving habitat suitability index models. Wildlife Soc Bull 25, 163-167.

Bryant FC, MM Kothmann, LB Merrill. 1980. Nutritive content of sheep, goat, and white-tailed deer diets on excellent condition rangeland in Texas. J Range Manage 33, 410-414.

Bryant FC, CA Taylor, LB Merrill. 1981. White-tailed deer diets from pastures in excellent and poor range condition. J Range Manage 34, 193-200. 
Buckland ST, AE Magurran, RE Green, RM Fewster. 2005. Monitoring change in biodiversity through composite indices. Philosophical Transactions Biological Sciences 360, 243-254

Bullock J. 1999. Plants. In: Ecological census techniques. A Handbook. Sutherland WJ (ed). Cambridge University Press, Cambridge, UK.

Codron DI, JA Lee-Thorp, M Sponheimer, J Codron. 2007. Nutritional content of savanna plant foods: Implications for browser/grazer models of ungulate diversification. Eur J Wildl Res 53, 100-111.

Cook RC, TR Stephenson, WL Myers, JG Cook, LA Shipley. 2007. Validating predictive models of nutritional condition for mule deer. J Wild Manage 71, 1934-1943

Deperno CS, JA Jenks, SI Griffin, LA Rice, KF Higgins. 2002. White-tailed deer habitats in the central black hills. J Range Manage 55, 242-252.

DeYoung RW, EC Hellgren, TE Fulbright, WF Robbins Jr., ID Humphreys. 2000. Modeling nutritional carrying capacity for translocated desert big horn sheep in Western Texas. Restoration Ecol 8, 57-65.

Galbraith JK, GW Mathison, RJ Hudson, TA McAllister, KJ Cheng. 1998. Intake, digestibility, methane and heat production in bison, wapiti and white-tailed deer. Can J Anim Sci 78, 681-691.

Gallina S. 1993. White-tailed deer and cattle diets in La Michilia, Durango, Mexico. J Range Manage 46, 487-492.

Gallina S, J Bello, CC Verteramo, C Delfin. 2010. Daytime bedsite selection by the texan white-tailed deer in xerophyllous brushland, North-Eastern Mexico. J Arid Environ 74, 373-377.

Galt D, F Molinar, J Navarro, J Joseph, J Holechek. 2000. Grazing capacity and stocking rate. Rangelands 22, 7-11.

Germaine SS, HL Germaine, SR Boe. 2004. Characteristics of mule deer day-bed and forage sites in current-condition and restoration-treated ponderosa pine forest. Wildl Soc Bull 32, 554-564.

Gordon IJ, AJ Hester, M Festa-Bianchet. 2004. The management of wild large herbivores to meet economic, conservation and environmental objectives. J Appl Ecol 41, 1021-1031.

Gotelli NJ. 2008. Logistic population growth. In: Gotelli NJ (ed). A primer of ecology. Sinauer Associates Inc., Sunderland, USA, Pp 25-48.

Griffith B, BA Youtie. 1988. Two devices for estimating foliage density and deer hiding cover. Wildl Soc Bull 16, 206-210.

Hobbs NT, DM Swift. 1985. Estimates of habitat carrying capacity incorporating explicit nutritional constraints. $J$ Wild Manage 49, 814-822.

Huerta-Martínez FM, JA Vázquez-García, E García-Moya, L LópezMata, H Vaquera-Huerta. 2004. Vegetation ordination at the Southern Chihuahuan desert (San Luis Potosi, Mexico). Plant Ecology 174, 79-87.

Jiang G, MA Jianzhang, M Zhang, P Stott. 2010. Multi-scale foraging habitat use and interactions by sympatric cervids in Northeastern China. J Wild Manage 74, 678-689.

Long ES, DR Diefenbach, CS Rosenberry, BD Wallingford, MD Grund. 2005. Forest cover influences dispersal distance of white-tailed deer. J Mammalogy 86, 623-629.

Mandujano S. 2007. Carrying capacity and potential production of ungulates for human use in a Mexican tropical dry forest. Biotropica 39, 519-24.

McCall TC, RD Brown, CL Bender. 1997. Comparison of techniques for determining the nutritional carrying capacity for white-tailed deer. J Range Manage 50, 33 -38.
McLeod SR. 1997. Is the concept of carrying capacity useful in variable environments? Oikos 79, 529-542.

Mertens D. 1987. Predicting intake and digestibility using mathematical models of ruminal function. J Anim Sci 64, 1458-1558.

Mysterud A, E Østbye. 1999. Cover as a habitat element for temperate ungulates: effects on habitat selection and demography. Wildl Soc Bull 27, 385-394.

Otto R, JM Fernández-Palacios, BO Krüsi. 2001. Variation in species composition and vegetation structure of succulent scrub on Tenerife in relation to environmental variation. $J$ Veg Sci 12, 237-248.

Peters EM, C Martorell, E Ezcurra. 2008. Nurse rocks are more important than nurse plants in determining the distribution and establishment of globose cacti (Mammillaria) in the Tehuacán Valley, Mexico. $J$ Arid Environ 72, 593-601.

Plata FX, S Ebergeny, JL Resendiz, O Villarreal, R Bárcena, JA Viccon, GD Mendoza. 2009. Palatabilidad y composición química de alimentos consumidos en cautiverio por el venado cola blanca de Yucatán (Odocoileus virginianus yucatanensis). Arch Med Vet 41, 123-129.

Plata FX, GD Mendoza, JA Viccon, R Bárcena, F Clemente. 2011a. Comparación de métodos basados en los requerimientos nutricionales y disponibilidad de biomasa para estimar la capacidad de carga para venado cola blanca. Arch Med Vet 43, 41-50.

Plata FX, Mendoza GD Viccon JA, Bárcena R, Sánchez FC, Villareal OA. 2011 . Adecuación y análisis de sensibilidad de un modelo para la estimación de la capacidad de carga del hábitat de venado cola blanca. Arch Med Vet 43, 267-275.

Pollock MT, DG Whittaker, S Demarais, RE Zaiglin.1994. Vegetation characteristics influencing site selection by male white-tailed deer in Texas. J Range Manage 47, 235-239.

Rzedowski J. 2006. Vegetación de México. Comisión Nacional para el Conocimiento y Uso de la Diversidad, México.

Rouvinen S, T Kuuluvainen. 2005. Tree diameter distributions in natural and managed old Pinus sylvestris-dominated forests. Forest Ecology and Management 208, 45-46.

Sall J, L Creighton, A Lehman. 1996. JMP Start Statistics: A guide to statistics and data analysis using JMP and JMP IN Software. Duxbury Press, CA, USA, Pp 171-184.

Sarkkola S, H Hökkä, R Laiho, J Päivänen, T Penttila. 2004. Stand structural dynamics on drained peatlands dominated by Scots pine. Forest Ecology and Management 206, 135-152.

Schuster JL. 1965. Estimating browse from twig and stem measurements. J Range Manage 18, 220-222

Short HL. 1986. Habitat suitability index models: White-tailed deer in the Gulf of Mexico and South Atlantic coastal plains. US Fish Wildlife Service Biological Report 82 (10.123), West Virginia,USA.

Stuth JW, WJ Sheffield. 2001. Determining carrying capacity for combinations of livestock, white-tailed deer and exotic ungulates. In: Wildlife Management Handbook. Texas A\&M University System, Texas, USA, Pp 5-12.

Telfer ES. 1969. Twig weight-diameter relationships for browse species. $J$ Wild Manage 33, 917-921.

Williamson SC, JK Detling, JL Dodd, MI Dyer. 1987. Nondestructive estimation of shortgrass aerial biomass. J Range Manage 40, 254-256.

Young CC. 1998. Defining the range: The development of carrying capacity in management practice. J History Biol 31, 61-83. 